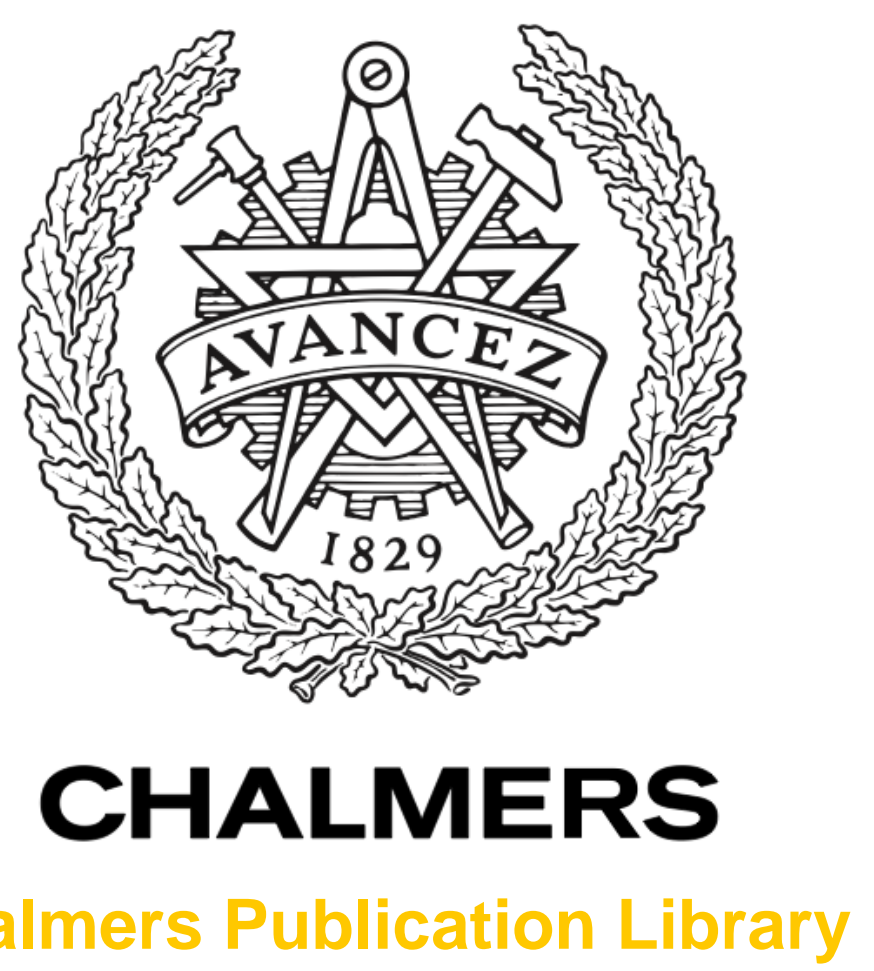

\title{
Forest Biomass Retrieval from BioSAR 2010 P-band SAR Data using a Regression- Based Model
}

This document has been downloaded from Chalmers Publication Library (CPL). It is the author's version of a work that was accepted for publication in:

\section{IEEE International Geoscience and Remote Sensing Symposium}

Citation for the published paper:

Blomberg, E. ; Soja, M. ; Ulander, L. (2015) "Forest Biomass Retrieval from BioSAR 2010

P-band SAR Data using a Regression-Based Model". IEEE International Geoscience and

Remote Sensing Symposium

Downloaded from: http://publications.lib.chalmers.se/publication/226251

Notice: Changes introduced as a result of publishing processes such as copy-editing and formatting may not be reflected in this document. For a definitive version of this work, please refer to the published source. Please note that access to the published version might require a subscription. 


\title{
FOREST BIOMASS RETRIEVAL FROM BIOSAR 2010 P-BAND SAR DATA USING A REGRESSION-BASED MODEL
}

\author{
E. Blomberg, M. J. Soja, and L. M. H. Ulander
}

\author{
Chalmers University of Technology, Göteborg, Sweden
}

\begin{abstract}
A model for retrieval of boreal forest biomass from polarimetric P-band SAR images developed using the BioSAR 2007 and BioSAR 2008 data sets is revisited and evaluated using data from BioSAR 2010. Incorporating the $\mathrm{HV}$ backscatter component as well as the $\mathrm{HH} / \mathrm{VV}$ ratio and the ground slope, the model is noteworthy as performing well when retrieving biomass values in Remningstorp using parameters trained in Krycklan. These two Swedish test sites are separated by $720 \mathrm{~km}$ and represent two different types of boreal forest as well varying topography. SAR images and biomass estimates obtained from Remningstorp in 2010 provides an opportunity to test the model further. This dataset results in a qualitative reproduction of the previous result, showing the expected changes due to forest management, but with a relative overestimation of biomass. A large part of this can be explained by the timing as the new acquisitions took place during early autumn instead of spring with the associated changes in moisture conditions.
\end{abstract}

Index Terms - Biomass retrieval, boreal forest, P-band, synthetic aperture radar (SAR), topographic correction

\section{INTRODUCTION}

BIOMASS, posed to become the first space-based P-band SAR as the seventh Earth Explorer mission, was selected by ESA after the BIOMASS feasibility study demonstrated that it is capable of providing global measurements of forest parameters such as biomass, biomass change and forest height. One of the results of this effort was the development of a regression model for the retrieval of boreal forest biomass from P-band SAR backscatter data [1]-[2].

Multiple campaigns to obtain airborne SAR and forest data were performed to support the BIOMASS proposal, including three in Swedish boreal forests; BioSAR 2007, 2008 and 2010 [3]-[5]. The model was shown to be capable of estimating biomass at one site, Remningstorp (BioSAR 2007), with a root-mean-square-error as low as $22-33 \%$, using model parameters estimated in the other test site, Krycklan (BioSAR 2008), with different types of forest and terrain, separated by $720 \mathrm{~km}$ from Remningstorp.

BioSAR 2010 provided updated and expanded forest data and SAR images for Remningstorp; however the processing of these data was not finished and they were therefore not included in [1]-[2]. This paper presents results from applying the model on this dataset and draws conclusions regarding the accuracy and variability of the biomass retrieval.

\section{THE MODEL}

The model gives the 10-logarithm of the biomass through linear regression of the HV backscatter coefficient $\left(\left[\gamma_{\mathrm{HV}}^{0}\right]_{\mathrm{dB}}\right.$, measured in decibels), the $\mathrm{HH} / \mathrm{VV}$ backscatter ratio

$\left(\left[\frac{\gamma_{\mathrm{HH}}^{\mathrm{O}}}{\gamma_{\mathrm{VV}}^{0}}\right]_{\mathrm{dB}}\right.$, measured in decibels) and product of the ground slope ( $u$, measured in radians), and the HH/VV ratio:

$$
\log _{10} B=a+b \cdot\left[\gamma_{\mathrm{HV}}^{0}\right]_{\mathrm{dB}}+c \cdot\left[\frac{\gamma_{\mathrm{HH}}^{0}}{\gamma_{\mathrm{VV}}^{0}}\right]_{\mathrm{dB}}+d \cdot u \cdot\left[\frac{\gamma_{\mathrm{HH}}^{0}}{\gamma_{\mathrm{VV}}^{0}}\right]_{\mathrm{dB}}
$$

This is motivated by the observation that $\mathrm{HV}$ backscatter is most consistently correlated with biomass and that taking the ratio of $\mathrm{HH}$ and $\mathrm{VV}$ will eliminate some of their common, biomass-independent contributions, such as moisture, forest structure (e.g. biomass consolidation [6]), surface roughness and topography. The ground slope is included to further decrease the topographic variations.

\section{BIOSAR DATA}

Remningstorp, the subject of BioSAR 2007 and 2010, is located in the south of Sweden at $58^{\circ} 28^{\prime} \mathrm{N}, 13^{\circ} 38^{\prime} \mathrm{E}$, has slopes up to $5^{\circ}$ (measured from a $50 \mathrm{~m} \times 50 \mathrm{~m} \mathrm{DEM}$ ) and a biomass $<300$ tons/ha consisting of Norway spruce, Scots pine and some birch. Krycklan, BioSAR 2008, is located in the north at $64^{\circ} 14^{\prime} \mathrm{N}, 19^{\circ} 46^{\prime} \mathrm{E}$, has slopes up to $19^{\circ}$ and biomass $<200$ tons/ha and is dominated by Norway spruce and Scots pine. The steeper slopes in Krycklan causes the across-site validation to perform much better when trained in Krycklan and validated in Remningstorp than vice versa, as the Krycklan terrain is not represented in Remningstorp data [2].

For both sites, there are $10 \mathrm{~m} \times 10 \mathrm{~m}$ biomass maps estimated using LiDAR acquisitions and a regular grid of in situ plots, as well as plot and stand level biomass estimates obtained using allometric equations and in situ sampled reference data. 

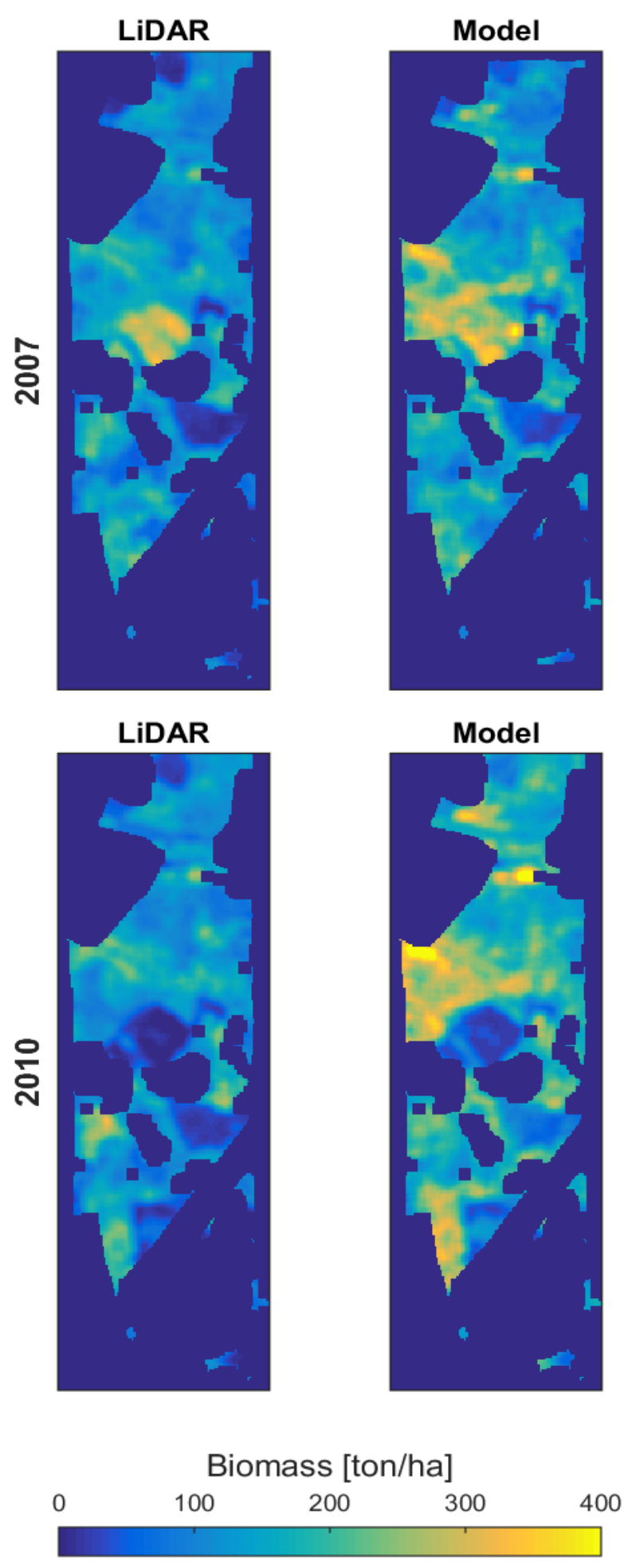

Figure 1. Biomass maps in ton/ha covering part of the Remningstorp forest test site in southern Sweden. The maps to the left are based on LiDAR measurements referenced to in situ data and the maps on the right are modeled, using the parameter values of Table 1 , from several polarimetric SETHI P-band SAR images acquired during BioSAR 2007 (top) and BioSAR 2010 (bottom).
The P-band SAR images available for Krycklan feature six different headings (of which four were used in [2]) acquired in the middle of October 2008. The images from Remningstorp are from two different headings in the beginning of March, April and May 2007 respectively (BioSAR 2007), with the BioSAR 2010 images following the same tracks plus one extra heading in September 2010.

Extending the analysis to the BioSAR 2010 data therefore results in the addition of temporal data, both a span of 3 years and a different season, as well as a new heading. It also enables a comparison of two different platforms as the SETHI system was used instead of ESAR which flew during BioSAR 2007 and 2008.

\section{RESULTS}

Table 1 contains the model parameter values obtained when using data from the Krycklan site [2], with Figure 1 displaying the model output from Remningstorp together with the corresponding LiDAR based biomass maps.

Table 1: Parameters estimated in Krycklan.

Polarimetric SAR images from both BioSAR campaigns were individually averaged and resampled to the same resolution and grid as the corresponding LiDAR-based biomass map and used together with interpolated slope values. Each set was then averaged together to a final $70 \mathrm{~m} \times$ $70 \mathrm{~m}$ resolution which is roughly the size of the smallest stand used in the training data set.

The model reproduces the features seen in the 2007 LiDAR measurements, with the possible exception of two high biomass areas in the center of the map. They are clearly visible in Figure 2, which displays the difference between the two biomass maps. A suggestion was made for a possible two part explanation in [2]: Small stands of large trees in the north western corner might contribute more to the biomass than evident from the heavily averaged LiDAR data. Closer to the center on the other hand is an area containing dense understory vegetation under a sparse canopy of pines, which might give a disproportionally strong HV signature leading to an overestimation.

Using the BioSAR 2010 backscatter images results in a general overestimation of the biomass which is especially marked for the areas already mentioned as well as a small area in the northern part and a large swath in the south. Another observation is that the felling of the high biomass stand in the center and a clearing in the south are easily distinguished both the biomass maps and the change map shown in figure 3.

The excessive biomass reported by the model for the most recent data is likely in mostly caused by a general increase in backscatter intensity. It was reported that the BioSAR 2010 values were up to $2 \mathrm{~dB}$ larger for $\mathrm{HV}$ and $3 \mathrm{~dB}$ larger for $\mathrm{HH}$ and $\mathrm{VV}$ compared to even the most recent 

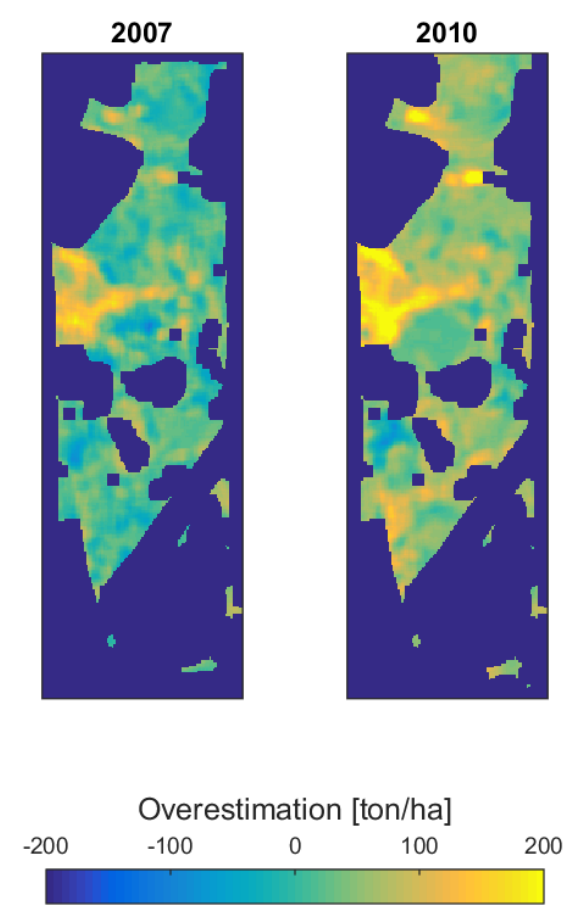

Figure 2. Difference between the model and the LiDAR data for BioSAR 2007 (left) and BioSAR 2010 (right), corresponding to the top and bottom row of Figure 1 respectively.

BioSAR 2007 acquisition. Such an increase was not deemed explainable considering only the differences between SETHI and ESAR and the small growth of biomass expected. It was therefore concluded that the variation of the moisture content of the forest and ground due to the different season was the most likely cause. The 2010 data might be more sensitive to these conditions as they represent an average of three directions for a single day while the 2007 map includes two directions during three different months.

A $2 \mathrm{~dB}$ decrease in the backscatter is enough to completely counteract the modeled overestimation and produce biomass values close to the biomass map.

\section{CONCLUSIONS}

The model performs as expected for the extended data set, reproducing the large changes due to forest harvest and the general features of the biomass map. There is a pronounced overestimation of the biomass which correlates well with a general increase seen in the backscatter values most likely caused by changing moisture conditions. This highlights the difficulty in compensating for variable water content also for this model.

\section{REFERENCES}

[1] L. M. H. Ulander, G. Sandberg, and M. J. Soja, "Biomass retrieval algorithm based on P-band BioSAR experiments of boreal forest," in Proc. IEEE IGARSS, Vancouver, Canada, Jul. 2011, pp. 4245-4248.
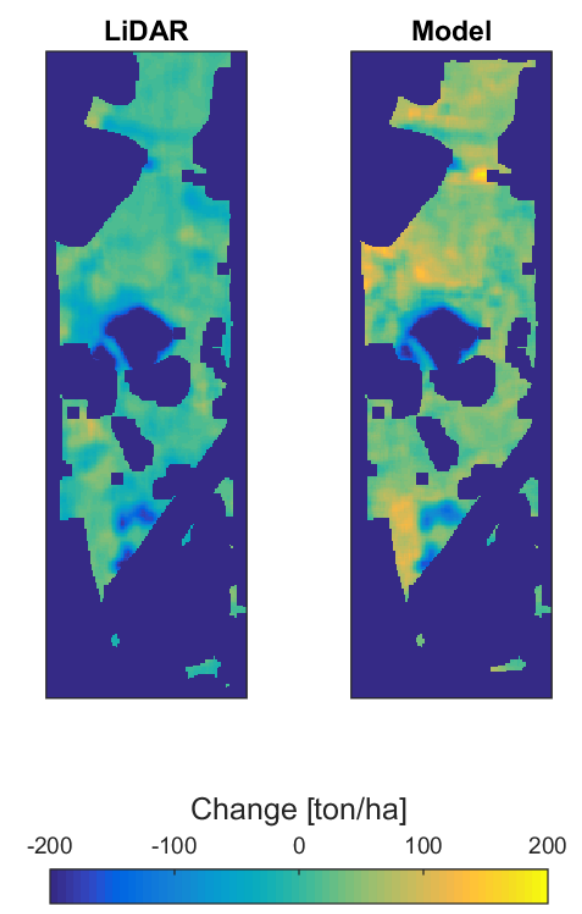

Figure 3. LiDAR biomass change (left) and the biomass change according to the model (right) from 2007 to 2010.

[2] M.J. Soja, G. Sandberg, G., and L.M.H. Ulander, Regressionbased Retrieval of Boreal Forest Biomass in Sloping Terrain using P-band SAR Backscatter Intensity Data, IEEE Trans. Geosci. Remote Sensing, vol. 51, no. 5, pp. 2646-2665, 2013

[3] I. Hajnsek, R. Scheiber, L. Ulander, A. Gustavsson, G. Sandberg, S. Tebaldini, A. M. Guarnieri, F. Rocca, F. Bombardini, and M. Pardini, "BIOSAR 2007 Technical Assistance for the Development of Airborne SAR and Geophysical Measurements During the BioSAR 2007 Experiment: Final Report Without Synthesis", 2008, ESA Contract No. 20755/07/NL/CB.

[4] I. Hajnsek, R. Scheiber, M. Keller, R. Horn, S. Lee, L. M. H. Ulander, A. Gustavsson, G. Sandberg, T. Le Toan, S. Tebaldini, A. M. Guarnieri, and F. Rocca, "BIOSAR 2008 Technical Assistance for the Development of Airborne SAR and Geophysical Measurements During the BioSAR 2008 Experiment: Final Report" 2009, ESA Contract No. 22052/08/NL/CT.

[5] L. M. H. Ulander, A. Gustavsson, B. Flood, D. Murdin, P. Dubois-Fernandez, X. Dupuis, G. Sandberg, M. J. Soja, L. E. B. Eriksson, J. E. S. Fransson, J. Holmgren and J. Wallerman, "BIOSAR 2010 Technical Assistance for the Development of Airborne SAR and Geophysical Measurements during the BioSAR 2010 Experiment: Final Report", 2011, ESA Contract No. 4000102285/10/NL/JA/ef.

[6] Smith-Jonforsen, G., Folkesson, K., Hallberg, B., and L. M. H. Ulander, Effects of Forest Biomass and Stand Consolidation on PBand Backscatter, IEEE Geoscience and Remote Sensing Letters, Vol. 4, No. 4, pp. 669-673, 2007 